\title{
Semantic Clustering
}

National Cancer Institute

\section{Source}

National Cancer Institute. Semantic Clustering. NCI Thesaurus. Code C162251.

The tendency to group items according to their semantic category. 